\title{
La esencia de la libertad en el enfrentamiento entre Jacobi y Schelling ${ }^{l}$
}

\author{
The essence of freedom in the confrontation \\ between Jacobi and Schelling
}

\author{
WILHELM. G. JACOBS \\ Ludwig-Maximilians-Universität München (Alemania) \\ Recibido: 08-03-2014 Aceptado definitivamente: 05-05-2014
}

RESUMEN

La disputa entre Jacobi y Schelling sobre los conceptos fundamentales de fundamento y causa a propósito de las cosas divinas (1811/1812) será analñizada con la intención de prinfundizar en la comrpensión de la libertad.

\section{PALABRAS CLAVES}

SCHELLING, JACOBI, LIBERTAD, VOLUNTAD, CAUSA Y FUNDAMENTO

\begin{abstract}
The in the dispute between Jacobi and Schelling about the divine things (1811/12) central notions of reason and cause are inquired into a sufficient understanding of liberty.

\section{KEY WORDS}

SCHELLING, JACOBI, FREEDOM, WILL, CAUSE, BASIS
\end{abstract}

\section{Suplemento 19 (2014)}

(C) Contrastes. Revista internacional de filosofía, pp. 61-73. ISSN: 1136-9922

Departamento de filosofía, Facultad de Filosofía y Letras, Universidad de Málaga

Campus de Teatinos, E-29071 Málaga (España)

1 Título original Das Wesen der Freiheit in der Auseinandersetzung zwischen Jacobi und Schelling. Traducción de Claudia Fernández Fernández y Alejandro Rojas Jiménez. 
EN SU CONMEMORACIÓN AL ESCRITO DE LAS COSAS DIVINAS... Schelling responde entre otras cosas a la distinción que hace Jacobi entre fundamento y causa, y de la nueva parte de aquel texto de las cosas divinas cita: «Se tiene sencillamente la opción, aceptada, de que el absoluto sea o fundamento o causa. Que sea fundamento y no causa es lo que afirma el naturalismo; que sea causa y no fundamento, el teísmo.» $\rangle^{2}$ Esta diferenciación obedece a aquel convencimiento, Jacobi lo subraya al comienzo, expuesto en el librito sobre Spinoza y en el $D a$ vid Hume. Klaus Hammacher señala que ambos, Jacobi y Schelling, trabajan «con la diferencia entre causa y fundamento ${ }^{3}$ y que toman para ello la «más significativa dirección sistemática de Jacobi». ${ }^{4}$

Birgit Sandkaulen se ha servido del uso que Jacobi hace de estos conceptos con el objeto de realizar una investigación detallada y fundamentada bajo el título de «Fundamento y causa. La crítica de la razón de Jacobi», a la que aquí se hace gustosamente referencia.Vista la importancia central de estos conceptos para Jacobi y, como se mostrará, también para Schelling, este trabajo se ceñirá a su aclaración. La dificultad estriba en que ambos filósofos utilizan los mismos términos, pero bajo ellos no entienden lo mismo. Estamos ante una dificultad notoria cuando lo que tiene que tematizarse es el debate sobre la noción de libertad en ambos filósofos, por lo que hay que dirigirse hacia el centro de controversia.

Puesto que Schelling se refiere a los conceptos de Jacobi, se aclarará en una primera parte la comprensión de Jacobi respecto de causa y fundamento. En una segunda parte se atenderá a la comprensión de Schelling de causa, en una tercera a la comprensión de éste respecto de la noción de fundamento, y por último y en cuarto lugar, a la autodeterminación.

\section{LA DISTINCIÓN DE JACOBI ENTRE FUNDAMENTO Y CAUSA}

Jacobi se pronuncia pronto acerca de los conceptos de fundamento y causa, en particular en el suplemento VII que agregó en 1789 a la segunda edición de su escrito Sobre la doctrina de Spinoza en las cartas al señor Moses Mendelssohn. La diferenciación de Jacobi es una parte de su argumentación contra la filosofía de Spinoza, justamente contra la noción de causalidad en éste. En la doctrina de Spinoza la sustancia queda determinada como causa sui y los modos en los que se vincula a los Modi como causa efficiens. La causa real es, pues, la forma en la que la causa sui se presenta en los Modi. Spinoza no conoce otra forma de causalidad; niega la causa finalis y con ella la libertad de la voluntad. Y esto no convencerá a Jacobi.

2 Denkmal 94. SW VIII, 71. SW no subrraya el no antes de causa. Cfr., JWA 3, 105.

3 Hammacher 1994, 140.

4 Hammacher1994, 141.

Suplemento 19 (2014) de Contrastes. Revista internacional de filosofía 
La essentia de la causa sui no puede ser comprendida según Spinoza, por existente. ${ }^{5}$ Le es adecuada su eternidad, que se determina como «ipsam existentiam, quatenus ex sola rei aeternae definitione necessario sequi concipitur.» ${ }^{6}$ Los modos no pueden ser pensados sin sustancia, pero ésta -de facto en todo casono puede ser sin los Modi, así concluye Jacobi interpretando a Spinoza ya en su primera edición de 1785 de su librito: «El llegar a ser (el movimiento de los Modi) no puede ser pensado ni como principio ni como ser» ${ }^{7}$ pues, se debe aclarar, cada Modus que pueda ser determinado como causa, es por su parte/por sí mismo efecto, y así tanto hacia delante como hacia atrás en/hacia el infinito.

La cita que se acaba de recoger ejemplifica la posición de Jacobi en el Suplemento VII. El movimiento de los Modi se determina para Spinoza sin excepción en virtud de la causa eficiente; ésta es por tanto, como dice Jacobi, la «forma general, invariable y eterna de las cosas individuales y su continuo cambio». ${ }^{8}$ Pero si cada causa tiene que ser comprendida como efecto de una causa precedente, entonces no hay comienzo, es decir: no hay pensada ninguna causa explicativa de todo el movimiento. Visto así, se deshace, como escribe Jacobi en 1811, la causalidad: «nada es necesario; no hay ningún (...) principio, (...) ningún comienzo absoluto y alzado». ${ }^{9}$

Si no se debe deshacer la causalidad, debe entenderse de acuerdo con Jacobi como un movimiento que se pone en marcha desde sí [unverursacht], una acción. ${ }^{10}$ Algo semejante piensa Spinoza, pues él niega la causa finalis, no consecuente. Jacobi objeta a Spinoza: «el movimiento no se habría iniciado nunca; así tampoco las cosas singulares podrían tener comienzo.» ${ }^{11} \mathrm{Si}$ no hay un primer comienzo, no se puede pensar cómo pudiera suceder una transmisión de fuerza (Kraftübertragung) real de una causa a un efecto. No se transforma pues nada con el tiempo; sucede en el fondo siempre lo mismo. Los Modi de Spinoza son a ojos de Jacobi «no sólo el origen de la eternidad; sino también [...] del concepto de razón, y al mismo tiempo de todo lo existente». ${ }^{12}$ Para la razón no hay según Jacobi ningún tiempo. Por el contrario: La causa efficiens sólo puede conocerse en el tiempo. Ésta mantiene «separados», ${ }^{13}$ como escribe Sandkaulen, causa y efecto. La relación entre fundamento y consecuencias no se conoce por experiencia, sino que es pensada, es decir, fuera del tiempo, o sea coincidentes. Jacobi remite para su aclaración a las relaciones geométricas: el concepto de triángu-

\author{
5 Cfr., Eth. P. I. def. 1. \\ 6 Eth. P. I. def. 8. \\ 7 JWA $1,1,93$. \\ 8 JWA $1,1,253$. \\ 9 JWA $3,110$. \\ 10 Cfr., Sandkaulen 2000, 185. \\ 11 JWA 1,1, 253. \\ 12 JWA $1,1,253$. \\ 13 Sandkaulen 2000, 189.
}


lo es fundamento y al mismo tiempo consecuencia de la suma de los ángulos. Pero Spinoza ha elevado erróneamente el concepto de experiencia a «concepto de razón». ${ }^{14}$ Con ello se confundiría, como formula Sandkaulen, la «relación entre una sucesión lógica o real». ${ }^{15}$

Sólo después de la afirmación de Jacobi puede ser experimentada la causalidad. Él sabe que nosotros observamos una sucesión externa, pero no la causalidad. Hasta aquí va en consonancia con David Hume, quién dará nombre además al diálogo, pertinente aquí, de 1787. Si la causalidad no se puede conocer por experiencia como algo externo, entonces sólo es experimentada en nosotros, quiero decir, a través de nuestra praxis: «inosotros también podemos actuar!». ${ }^{16}$ Al actuar nos sentimos como una causa libre, que como tal sufre también resistencia. Por consiguiente, se distinguen según Jacobi causa y fundamento, pues la noción de causa es «un concepto de experiencia que debemos al hecho de que somos seres conscientes de nuestra causalidad y pasividad, y que no deriva de los conceptos puramente idealistas acerca del fundamento, en esos mismos en los que se deshace. $\rangle^{17}$ De esto modo la noción de causa, como señala Siegbert Peetz, pertenece finalmente a una filosofía práctica, y la de fundamento a una filosofía teórica. ${ }^{18}$

La noción de causa debe ser, en tanto que Jacobi la piensa desde la acción, suficientemente aclarada. Como actuante [handelnd] pensamos en una persona que es capaz de, desde sí, modificar algo del mundo sensible. La acción del autor es vista como un acontecimiento en el tiempo. Jacobi lo dice expresamente: «mediante la noción de causa se sitúa una acción necesariamente en el tiempo de forma inamovible; pues una acción que no sucediera en el tiempo es un absurdo». ${ }^{19}$ Jacobi apela a la experiencia propia que tenemos al sabernos libres en el actuar [handeln]. Nos [re]conocemos [wissen] como personas responsables en la experiencia de nuestra existencia, identidad y autonomía. ${ }^{20} \mathrm{Al}$ entender Jacobi la causa como acción, entonces, está «eo ipso en relación con aquello que ha sido causado»». ${ }^{21}$ Con ello el que actúa [der Handelnde] tiene a la vista, al comienzo de una acción, el final, la meta. Sandkaulen cita la Kladde VII de Jacobi: «Causa es inicio [Anfang] -inicio es acción- de la causa final [Endursache], donde el inicio está al final, o viene del final.»» ${ }^{22}$ Eso, que se debe lograr, el

14 JWA 1,1, 253.

15 Sandkaulen 2000, 175.

16 JWA 2,1, 53.

17 JWA 1,1, 256.

18 Cfr., Peetz 1995, 168.

19 JWA 1,1, 257.

20 Cfr., Sandkaulen 2000, 191s.

21 Sandkaulen 2000, 195.

22 Sandkaulen 2000, 199. Kladde VII, p. 12.

Suplemento 19 (2014) de Contrastes. Revista internacional de filosofía 
Telos, determina la acción inicial. En la acción están unidas por lo tanto causa efficiens y causa finalis.

La finalidad es puesta por un ser [Wesen] que debía estar ya siendo Un ser. Este ser no sólo tiene que constituirse como Un ser, sino que antes tiene que encontrarse como tal, como dice en las Cosas divinas, «a través de una inmediata [...] impresión, no como un conocimiento [mediado WJ]; es ese Uno y lo mismo, [...] porque la sabiduría espiritual inmediata del espíritu es inseparable de la mismidad, de la sustantividad». ${ }^{23}$

En la nota correspondiente Jacobi designa a este ser como una persona que describe como «ser en sí», «ser mismo», «causa-misma» y «extratemporal», que está «en posesión de una conciencia extratemporal meramente interna». ${ }^{24}$ Sandkaulen explica la falta de temporalidad de la persona como sigue: «si el que actúa [Handelnde] estuviera sometido él mismo enteramente al tiempo, sería también sólo un eslabón más en la cadena de relaciones naturales y por consiguiente no aquel que podría empezar algo de modo incondicionado.» ${ }^{25}$ Puesto que la persona, sin embargo, actúa, no es temporal, «ciertamente [...] eso extratemporal es lo que nosotros designamos como voluntad e [...] intención y ejecución, autoapropiación y arrepentimiento [...] y conciencia humana». ${ }^{26}$ Lo extratemporal de la persona lo entiende Jacobi también como un componente integral de la acción temporal. Jacobi entiende la libertad desde la acción, y lleva toda la razón al afirmar que ésta no se puede pensar sin el tiempo.

Por mediación de la distinción entre fundamento y causa Jacobi critica que Spinoza discuta el hecho mismo de la libertad humana, y que en lugar de un Dios vivo y libre ponga la interna necesidad de la sustancia. La doctrina del Dios libre la denomina Jacobi teísmo, la otra naturalismo. Ambas afirman que lo infinito es previamente pensado como «un absoluto no condicionado». ${ }^{27} \mathrm{La}$ diferencia la ve Jacobi en que en el naturalismo Dios es fundamento, mientras que en el teísmo es afirmado como causa. El naturalismo afirma, dicho con las palabras de Jacobi, «que lo incondicionado o absoluto que presupone la razón, es sólo el sustrato condicionado, lo Uno del universo» ${ }^{28}$ El teísmo sitúa por el contrario previo a la naturaleza a un Dios libre en todos los aspectos. Nuevamente en palabras de Jacobi: «esto incondicionado o absoluto sería una causa autoconscientemente libre, causa análoga a la voluntad racional, una inteligencia suprema que actúa según el fin». ${ }^{29}$ La diferencia la señala Jacobi claramente en que o se piensa una cosa o la otra, Dios como fundamento o como causa. Tal y

\author{
23 JWA 3, $26 \mathrm{~s}$. \\ 24 JWA 3, 27. \\ 25 Sandkaulen 2000, 200. \\ 26 JWA 3, 108. \\ 27 JWA 3, 105. \\ 28 JWA 3, 108. \\ 29 JWA 3, 108.
}

Suplemento 19 (2014) de Contrastes. Revista internacional de filosofía 
como lo afirma es consecuente con ello. Él se entiende como teísta, piensa pues a Dios como causa, y de este modo libertad. El naturalismo es por ello ateísmo $\mathrm{y}$, aún cuando no se menciona el nombre de Schelling, también se refiere a su filosofía como ateísmo.

\section{LA COMPRENSIÓN DE CAUSA DE SCHELLING}

Schelling no admite el presupuesto de la crítica de Jacobi de que Dios -y en definitiva la libertad- sea fundamento o causa; él sostiene que ambos (Dios y la libertad) han de ser tanto fundamento como causa. Y ello porque aunque él coincide con Jacobi en que Dios es pensado como causa, argumenta sin embargo que hay que pensarlo también como fundamento. Esto no significa desde luego que piense la noción de causa como lo hace Jacobi, todo lo contario. Si se pretende fijar con precisión las diferencias entre ambos filósofos, se ha aclarar también la diferencia respecto de la noción de causa.

Una primera diferencia la sitúa Jacobi en la comprensión de la historia del pensamiento. El pensamiento racional, que se sirve conscientemente de la categoría de la causalidad, es, como sabe Jacobi, un primer nivel del desarrollo humano. La racionalidad, como nos resulta conocido, es algo que se ha ido ganando. Se «construye un mundo racional [...]. Hacemos un universo, en el que nosotros vamos despedazando, y adecuándolo a nuestras facultades, todo el mundo real y diverso de imágenes, ideas y palabras» ${ }^{30}$ La humanidad anteriormente había comprendido por el contrario los procesos naturales como acciones, y así los habían comprendido mediante la noción de causa. Sólo la racionalización posterior hace posible el naturalismo y que se dejen ver desde esta perspectiva como una abstracción inadmisible.

Schelling conocía ya en 1793 la diferencia entre el mundo antiguo, mítico, y el mundo nuevo, racional. ${ }^{31}$ Tenía claro que, como estudiante de teología, debía buscar comprender los textos ancestrales como textos con sentido y verdaderos, pero que estaba claro que estos textos sólo se entienden, y se reconoce en ellos sentido y comprensión, bajo aquella condición de que la racionalidad sea la misma para todos, en los tiempos de antes y los de después. Sin embargo, inicialmente no se reconocía una misma racionalidad, tanto que no podían ser considerados racionales los hombres primitivos en el mismo sentido en el que serán considerados después. Ciertamente en el mundo antiguo muchas veces lo que no se comprendía se aceptaba como acciones de los Dioses u otros seres. Pero también el hombre mítico debe, para poder actuar él por sí mismo, presuponer una causa natural constante y regular, es decir ordenada, aún cuando no reflexione sobre esta regularidad. Por eso el punto de vista de Jacobi de que la racionalidad

30 JWA $1,1,249$. 246.

31 Ueber Mythen, historische Sagen und Philosopheme der ältesten Welt. AA I,1, 193- 
sea un fruto posterior, no es ningún argumento a favor de una vigencia limitada de la racionaldiad. La racionalidad no podría ser un fruto posterior si no estuviera ya antes de un modo seminal, naturalmente sin desarrollar. Sin esta condición no habría una continuidad pensable entre el mundo antiguo y el moderno, sino una ruptura. La racionalidad debe, pues, ya sea de modo inconsciente o consciente, ser supuesta para todas las épocas.

Según Jacobi «se [la] sitúa con la noción de causa (...) en el tiempo de un modo inamovible». ${ }^{32}$ Esta tesis necesita según Schelling, que aquí sigue a Kant, de la diferenciación -de una, que claramente va más allá de la que acabamos de mencionar. La categoría de la casualidad de Kant es la forma del juicio hipotético. La relación del pensar es en este juicio la «del fundamento hacia sus consecuencias» ${ }^{33}$ en tanto que el tema es el pensar como tal, aún sin discurso. Sólo en caso de que la categoría se una con el tiempo, es decir en el esquema, puede estar el habla y tiene que estar según Kant una causalidad consolidada en el tiempo. Porque la categoría es la forma del pensar, pero el pensar no nace en el tiempo, se une en el esquema con el tiempo, por eso es para Kant, como también para Schelling, pensable la causalidad [Kausalität] y también la causalidad originaria [Ursächlichkeit] sin conexión al tiempo. Sobre eso más inmediatamente.

Lo que aparece en el tiempo es lo que está sujeto a la causalidad natural, tanto si lo podemos comprender como si no. En tanto son fenómenos humanos, y esto somos nosotros, cuando se nos aparece nuestra vida interior, nos parecen de acuerdo con las categorías, también la de causalidad. Por eso Kant afirma que si fuera conocido cada móvil de la conducta de un hombre, contaría su «acción con un futuro cierto, tanto como la luna o el eclipse solar». ${ }^{34}$ Lo que se nos hace consciente como nuestra alma, el caso ya citado de Jacobi sobre el autoaprobación y el arrepentimiento, es según Kan fenómeno, por ello está determinado causalmente, y se nos muestra sin libertad. La libertad es pensable sólo como exterior al tiempo. Ella no es sólo la experiencia de la decisión o la acción, más bien está en cada caso como aquella máxima fundamental según la cual nosotros nos disponemos hacia el bien o el mal.

La doctrina de Kant al respecto es asumida por Schelling; no sólo recordamos el escrito sobre la libertad, en el que se habla de la voluntad «eterna e independiente del tiempo». ${ }^{35} \mathrm{La}$ voluntad es entendida por Schelling como autodeterminación de sí misma, y de este modo como causalidad incausada. Ésta él la pueda entender con Kant sólo de un modo atemporal. Claramente, lo que Schelling tiene que diferenciar con Kant, son dos cosas: por un lado la causalidad en los fenómenos, la causalidad natural, y por otro lado la causalidad de la volun-

32 JWA $1,1,257$.

$33 \mathrm{KrV}$ A 73, B 98. Vgl. Logik § 25, in: Schriften IX, 105.

$34 \mathrm{KpV} 177$

35 SW VII, 350. 
tad, una causalidad ligada [gebunde] y otra libre. Donde Schelling, Kant y Jacobi coinciden es en que la noción de causa es un poder de inicio incausado. Pero este poder de inicio debe ser entendido como intemporal según Schelling, continuando a Kant y en contraposición a Jacobi.

Por consiguiente, tanto para Kant como para Schelling la acción que determina la vida de los hombres no pertenece al tiempo, sino a la eternidad. Schelling previene de un malentendido: esta acción «precede a la vida [...] no en el tiempo, sino a través del tiempo (sin conmoverse de él) durante el tiempo, como una acción de la naturaleza tras la acción eterna». ${ }^{36} \mathrm{La}$ acción va así a través del tiempo, más o menos como la voluntad que guía un matrimonio, a través del tiempo, a menudo a lo largo de las décadas. La voluntad se manifiesta en las decisiones y acciones individuales, pero está como una cosa en sí, o dicho schellingianamente como acción eterna fundamentando a ésta.

Esta voluntad no puede ser presentada como la meta de la acción, más bien se diferencia de la voluntad en una abundancia de metas de acciones individuales. La voluntad tiene que ser pensada como máxima, como norma de un orden superior. Schelling piensa la voluntad, con Kant y Jacobi, como un inicio incausado, pero no sigue a Jacobi al pensarla como una experiencia inmediata en el tiempo. Por el contrario, la voluntad se mantiene a través del tiempo, sitúa fundando lo que llega a ser individual.

La voluntad se ejecuta a sí misma como autodeterminación. En esto coincide Schelling con ambos autores, que la Aseität de la voluntad, y a través de ella, se ubica la voluntad, que a través del tiempo se mantiene como fundamento. Inmediatamente después de la frase introductora de la conferencia sobre Jacobi de Schelling acerca del fundamento y la causa se recoge la siguiente cita: «que aquí no hay sencillamente ninguna elección, el absoluto es tanto fundamento como causa y tiene que ser pensado como ambas cosas». ${ }^{37}$ Lo que vale para lo absoluto, vale además para la libertad. La voluntad-causa, es decir el inicio incausado que se determina, está en la autodeterminación, fundamento para ser eso a lo que la voluntad se determina causalmente. Lo que Schelling entiende bajo fundamento será expuesto ahora con más precisión.

\section{LA COMPRENSIÓN DE FUNDAMENTO DE SCHELLING}

La autodeterminación sitúa en la voluntad, sea divina o humana, una diferencia, es decir la de lo determinado y lo determinable. Esta diferenciación la conoce Schelling desde su Timeo -manuscrito de 1794 del Filebo de Platón. Ya entonces situaba al mismo tiempo lo apeiron y el concepto de materia ${ }^{38}$ él piensa esta completa indeterminación, o carencia de forma, de la que no cabe ninguna

36 Freiheitsschrift SW VII, $385 \mathrm{~s}$.

37 Denkmal 94, SW VIII, 71.

38 Cfr., Timaeus 59

Suplemento 19 (2014) de Contrastes. Revista internacional de filosofía 
esencia objetiva, como el momento de un existente. Pero esta materia de la determinación es pensada por Schelling como voluntad, es decir como una voluntad sin determinación. Pero puesto que una voluntad quiere llevar su ser hacia una meta, puesto que tiene una determinación, no estaríamos ante una voluntad como tal, una efectiva voluntad. Una voluntad tal es una voluntad que no quiere nada. Pues esta voluntad no puede ser pensada como efectiva, sino como la materia en tanto que momento o potencia de una voluntad real. Schelling describe, en el escrito sobre la libertad, esta voluntad carente de determinación como «un mar revuelto y ondulante, idéntico a la materia de Platón». ${ }^{39}$

Schelling concibe la voluntad como autodeterminación. Sólo tiene sentido hablar de ella en la medida en que algo indeterminado puede llegar a ser determinado. Esto indeterminado pone necesariamente la determinación como su fundamento [Grunde]; es el cimiento [Fundament] o, como también dice Schelling, la base [Basis] de la determinación. Puesto que Schelling piensa así, llega a emplear incluso la palabra sustancia y sujeto en su sentido etimológico originario que se corresponde significativamente con fundamento. $Y$ aquí, la noción de fundamento de Schelling, no sólo se diferencia claramente del modelo platónico, sino también del de Jacobi. Fundamento es para estos, como se ha indicado previamente, un concepto teórico que explicita la relación lógica. Por el contrario Schelling concibe este concepto de un modo práctico y designa como fundamento lo que mantiene algo en pie, aquello desde donde algo puede desarrollarse. Con ello el discurso de Schelling sobre el fundamento es doble. Por un lado es el fundamento determinable para lo determinado; ese tiene que ser libre, puesto que es pensado como causa en el sentido del Filebo. Pero también resulta que es el fundamento determinado en el que se sostiene, como voluntad determinada, la ejecución de la voluntad en el tiempo.

Porque Schelling piensa la voluntad como autodeterminación, señala en su Conmemoración el rechazo de Jacobi a aquellos filósofos que dejan «que se produzca lo perfecto desde lo imperfecto», ${ }^{40}$ volviendo a su lado de la cuestión. Lo imperfecto llega a ser sólo a través de lo determinado perfecto, reposa su fundamento en él. Jacobi no piensa esta relación, por eso no puede pensar ninguna autodeterminación, es decir, ninguna libertad (amplia). Pero porque lo determinable no es enemigo de una autodeterminación, sino algo que pertenece a lo determinable mismo, tiene que ser pensado esto determinable como voluntad indeterminada. Schelling designa esta voluntad como la naturaleza en Dios.

Esta naturaleza es de este modo voluntad carente de determinación. No tiene nada que pudiera querer. Se vuelve sobre sí misma sin ningún Telos. No puede siquiera quererse a sí misma, ni ser voluntad propia. Para ello, para quererse, necesita la determinación, el Telos. Determinación es decisión y con ello con-

39 SW VII, 360.

40 Denkmal 78. SW VIII, 62.

Suplemento 19 (2014) de Contrastes. Revista internacional de filosofía 
cepto, éste está en la voluntad general. No en vano designa Schelling la voluntad general también como logos, porque una palabra siempre rebasa la expresión. La voluntad carente de determinación, de este modo, llega a ser voluntad propia en confrontación con la voluntad general, en la que la voluntad general va más allá de lo propio. Porque sólo como voluntad propia responde oponiéndose la voluntad al logos, no se quiere de un modo puramente espiritual, sino también de un modo natural. No puede ir más allá de sí; lo podría si fuera logos. Por eso quiere ser un ser único, quiere su autoconservación. Sin el querer natural de sí mismo no sería posible por el contrario la voluntad general; ella necesita de la voluntad propia, porque ella no puede ir más allá de sí si no es algo propio. Ambas formas de la voluntad están, como Thomas Buchheim escribió, aunque en un contexto algo diferente, mutuamente acopladas.

La determinación es pues sólo mediante la voluntad general, que hace posible el logos. Esta voluntad se diferencia con respecto a la voluntad propia. Ésta no se puede diferenciar de sí, porque es la naturaleza no espiritual. Se expresa en el hombre como apetencia o desgana, lo que en sí no es nada malo. Solo llega a ser malo cuando la voluntad propia anula a la voluntad general. Pero también se convierte en una suerte de apetencia de indiferenciables deseos concretos, o de diferentes impulsos de autoconservación.

Estamos unidos a la naturaleza en su totalidad a través de la apetencia y su correspondiente dolor. Si estamos unidos con ella mediante nuestros sentimientos, estamos conectados a la naturaleza mediante nuestros impulsos naturales. Pero esta naturaleza es nuevamente sólo gracias a su contrario, la voluntad general. Aquí se acerca Schelling -esto debe estar dicho en el jubileo en honor de Fichte- a aquel dicho acerca de lo material del deber.

La naturaleza, en la que vivimos, está regulada por aquel. También para ella vale eso de que la determinación es de algo determinable, y así permanece siempre «aún en el fundamento lo carente de regla, que podría una vez más irrumpir, y aparecer de la nada, no siendo el orden y forma lo originario, sino como irrumpiendo hacia lo ordenado como una inicial carencia de regla. Ese es la inconcebible base de la realidad en las cosas, [...], lo que [...] permanece eternamente en el fundamento». ${ }^{41}$

Jacobi observa que la determinación exige un algo determinable en él mismo, es decir, un existente, un fundamento de su existencia, ${ }^{42} \mathrm{o}$ con otras palabras, tiene que tener en sí una naturaleza. Pero la autodeterminación, en la concepción de Schelling, es comprensible a través de la ejecución de la libertad, es decir, de la voluntad. "Querer es el ser originario», ${ }^{43}$ la autodeterminación es pues la concepción fundamental del ser. Lo cual alberga aún un problema que

41 Freiheitsschrift SW VII, $359 \mathrm{~s}$.

42 Cfr., Freiheitsschrift SW VII, 358.

43 Freiheitsschrift SW VII, 350. 
hasta el momento hemos ido rodeando, pero al que ya es momento de encaminarnos.

\section{AUTODETERMINACIÓN}

En una determinación no es pensable sólo una diferencia entre determinante y determinado, sino también entre los estados de indeterminación y determinabilidad. Se llega a pensar así un cambio del determinante en sí mismo, con el que enfrentamos dos estados opuestos. No habría ningún problema en ello si Schelling no hubiera situado con Kant la voluntad fuera del tiempo en la eternidad. Pero tenemos ahora a la voluntad situada fuera del tiempo. El tiempo es -según kant en todo caso- la única condición, para hacer que se dé «la posibilidad de un cambio, es decir de una unión de predicados opuestos [...] pensados en uno y el mismo objeto». ${ }^{44}$ El tipo de objeto válido en este caso sólo puede ser el de un sujeto. ¿Pero no tiene Jacobi el triunfo en la mano al atribuir la causalidad a la acción que se consolida en el tiempo?

No es necesario indicar que Kant aquí habla de la filosofía teorética. Se tiene que atribuir en la autodeterminación dos predicados del mismo sujeto y opuestos, es decir, que se mantienen separados sin causar problemas. Si esto es así, no se puede concebir la eternidad de Schelling como una «indiferencia», ${ }^{45}$ tal y como dice Fichte en las Instrucciones para una vida feliz respecto del Dios eterno. Pues la eternidad no puede ser pensada como invariable y pura indiferencia. Wolfgang Wieland ${ }^{46}$ distingue un tiempo impropio, la forma de la experiencia de Kant, y un tiempo propio. El primero sería un concepto de la filosofía teórica y el segundo de la filosofía práctica. Este sería el de la eternidad.

Si no debe ser pensado desde la filosofía teórica correspondiente al tiempo de la forma de la experiencia, que antecede en todo momento, que aún no ha sido a través del tiempo determinado con la categoría de la causalidad en el esquema causa, pues esta eternidad puede ser aquí un tiempo pensado o una eternidad sin medida en la que ningún esquema fija un orden. La voluntad no puede por ello estar en el tiempo. Pero tampoco puede ser pensada fuera del tiempo, si se debe evitar la contradicción. Sólo nos queda que la voluntad sea pensada de tal manera que, porque quiere ser efectiva, quiera inevitablemente la contradicción que su ejecución haría imposible de evitar.

Si pensamos la ejecución de la voluntad, si la penamos como actividad, entonces tiene que ser pensada como inicio. Inicio quiere decir causa incausada en la que ésta se determina y en la que acaba con la indeterminabilidad, en palabras de las Edades del Mundo: se sitúa en el pasado. Pero porque la determinación es determinación hacia algo, por ello el Telos determina el inicio, situándose

$44 \mathrm{KrV}$ B 48.

45 GA I,9, 90.

46 Wieland 1956, Cfr., por ejemlpo p. 64. 
igualmente el futuro. Con ello se trata del tiempo de la actividad, de la voluntad según está aquí entendida, y el presente es el modo primario, desde donde se erigen pasado y futuro.

Para Jacobi la causa está situada sólidamente en el tiempo, para Schelling funda el tiempo, y así reposa en ella, como expresa la palabra funda, la actividad de la voluntad.

Si después el hombre no vive en el tiempo, sino que genera, está por ello, como afirma Schelling, «fuera de todo el tiempo». ${ }^{47}$ Él quiere, decidirse, separarse de su pasado. Esto se puede entender psicológicamente, pero aquí está pensado de modo fundamental. Él rompe con su indecisión, lo que le capacita para su libre decisión. La indecisión no es sin embargo lo primero. Ella sólo llega a ser a través de la decisión. La decisión de una o más posibilidades separa unas de otras, de las que se han decidido. Esto vale kantianamente si hablamos de las maximas fundamentlaes de nuesta vida, o de la ley como ley, es decir, que hay que seguir o no, sin excepción. Se entiende que una decisión no es experimentable en el tiempo; lo que es experimentable puede ser como mucho la acción tal que conviene al carácter del hombre. El punto de vista de Kant y Schelling permite comprender el tiempo vital como un desarrollo progresivo de decisiones que son entendidas como manifestaciones de una acción eterna.

Por eso no es tampoco ninguna palabrería mítica cuando Schelling dice que esta acción se produce «junto con la primera creación (y al mismo tiempo como un acto distinto de aquel)». ${ }^{48}$ Palabrería mítica significaría que el hecho que somos, hubiera tenido lugar hace millones de años. Porque se sitúa esta acción en la forma de la experiencia del tiempo y porque con ello la intención de Schelling se pierde completamente. Esta primera creación es la creación originaria que no cae en el tiempo, sino que lo funda. Ella atraviesa el tiempo. En éste el hombre es querido como libre, es decir, un ser que tiene que crearse originariamente. La acción originaria del hombre rige «hasta en el inicio de la creación, por eso está fuera de todo lo creado, es un inicio libre y en sí mimo eterno». ${ }^{49} \mathrm{La}$ acción rige por eso al inicio, porque el hombre se crea con su acción y por ello está fuera de lo creado, de aquello que no es una criatura espiritual. El hombre es como Dios, sin ser Dios; pues él rige al inicio, no lo precede. Él se crea al decidir, pero las posibilidades de la decisión no las crea él.

Schelling piensa la libertad como autodeterminación radical. Para Jacobi la libertad es una conciencia inmediata dada -él dice sentimiento- indubitable. Esto los diferencia. Jacobi no se deja disuadir de su sentimiento. Lo que parece reconocer con respeto Schelling cuando escribe al inicio del escrito de la libertad que: «las investigaciones filosóficas sobre la esencia de la libertad humana pue-

47 Freiheitsschrift SW VII, 385.

48 Freiheitsschrift SW VII, 385.

49 Freiheitsschrift SW VII, 386.

Suplemento 19 (2014) de Contrastes. Revista internacional de filosofía 
den empezar en parte por el concepto correcto en el que el hecho de la libertad, es el mismo sentimiento inmediato grabado en todos (si bien de ningún modo se sitúa más en la superficie), que para ser expresado sólo con palabras, no requiere de una nitidez mayor que la cotidiana, ni se exige una profundidad del sentido»; estas palabras se pueden leer como un reconocimiento no sólo del pensamiento, sino también de la persona de Jacobi. El auténtico problema lo formula Schelling inmediatamente a continuación: «en parte [las investigaciones sobre la libertad] pueden afectar la conexión de esta noción con todo un punto de vista científico»». ${ }^{50}$ Este problema más allá de su naturaleza y del pensamiento de Jacobi.

Estoy contento de no estar en la posición del arcángel Miguel; yo he ponderado sólo argumentos. Por lo que a los argumentos respecta, me parece que la defensa de la libertad de Jacobi, la de la afirmación y el recurso de una conciencia inmediata, es dejada atrás por el modo en el que la fundamenta el filosofar de Schelling. La filosofía del sentimiento de Jacobi sólo se puede defender con dificultades en el discurso actual de la psicología y de la investigación cerebral, mientras que aquellas de Kant y Schelling no se ven afectados en absoluto.

WiLhelm G. JACOBS es Professor en la Ludwig-Maximilans-Universität München,

Lineas de investigación:

Idealismo alemán, especialmente Schelling y Fichte, sus críticos como Jacobi y sus antecedentes.

Algunas publicaciones recientes:

Johnann Gottlieb Fichte. Eine Biographie, Insel, Berlin, 2012

Schelling lesen, legenda 3, Stuttgart-Bad Cannstatt 2004

Dirección electrónica: jacobs.wg@gmx.de

50 SW VII, 336.

Suplemento 19 (2014) de Contrastes. Revista internacional de filosofía 